\title{
Astrometric comparisons of quasar catalogues (Research Note)
}

\author{
J. Souchay ${ }^{1}$, S. B. Lambert ${ }^{1}$, A. H. Andrei ${ }^{1,2}$, S. Bouquillon ${ }^{1}$, C. Barache ${ }^{1}$, and C. Le Poncin-Lafitte ${ }^{1,3}$ \\ ${ }^{1}$ Observatoire de Paris, Systèmes de Référence Temps Espace (SYRTE), CNRS/UMR8630, Paris, France \\ e-mail: Jean. Souchay@obspm.fr \\ 2 Observatório Nacional/MCT and Observatório do Valongo/UFRJ, Rio de Janeiro, Brasil \\ 3 Technische Universität Dresden, Institut für Planetare Geodäsie, Lohrmann Observatorium, Dresden, Germany
}

Received 7 August 2007 / Accepted 1 April 2008

\begin{abstract}
Context. Large surveys, such as the Sloan Digital Sky Survey (SDSS) and the 2-degree Field (2QZ) considerably increased the number of recorded quasars at optical wavelengths. At the same time, the densification of very long baseline interferometry observations of extragalactic radio sources enabled an increase in the accuracy of the the quasi-inertial radio reference frame that realizes the International Celestial Reference System (ICRS).

Aims. In the perspective of the realization of an accurate optical counterpart to the ICRF, we investigate the astrometric properties of quasars recorded by several surveys, both at radio and optical wavelengths.

Methods. Cross-identifications of quasars are made between catalogues, both at optical (SDSS vs. 2QZ) and radio wavelengths (VLBA Calibrator Survey vs. SDSS). Equatorial coordinates of the quasars are compared to evaluate the astrometric quality of the catalogues.

Results. We find more than 2000 objects in common between SDSS and 2QZ. We show that differences in position never exceed 1", with a root mean square of $0.2^{\prime \prime}$. The agreement in redshift determinations is good with a typical dispersion of 0.05 root mean square. Similar comparisons between the SDSS and the VLBA Calibrator Survey find 831 cross-correlated sources within a $0.23^{\prime \prime}$ search radius. Systematic errors, likely due to the partial sky coverage of the SDSS, are detected in the coordinate differences, which produces to a rotation of the optical frame, with respect to the radio counterpart, at the level of 13 mas. Thus both the SDSS and the 2QZ can provide invaluable information for the accurate determination of the ICRS at the fainter end of the optical domain. We finally point out that the highest probability of finding radio loud quasars involves searching in the redder regions of the color-color space.
\end{abstract}

Key words. astrometry - reference systems - astronomical data bases: miscellaneous - quasars: general

\section{Introduction}

Many fields of research in astronomy require the most accurate realization of an inertial celestial reference system. Celestial reference frames were first built using optical telescopes with an accuracy of typically 0.1 arcsec. The development of very long baseline interferometry (VLBI), with observations at centimeter wavelengths, offered the milliarc second (mas) accuracy in the late 1980s. The VLBI-based fundamental International Celestial Reference Frame (ICRF, Ma et al. 1998) was adopted at the International Astronomical Union XXIII General Assembly, Kyoto, Japan, in 1997. The ICRF axes are defined by the radio positions of 212 defining compact extragalactic objects, obtained from the analysis of VLBI observations until 1995. These axes are non-rotating with respect to the distant universe, and show little or no time dependency as well as a good time stability of $\sim 0.02$ mas.

The maintenance and the development of the radio reference frame obviously remains crucial for, e.g., geodetic VLBI activities. Since an increasing number of optical observations of quasars are provided by ground-based and space surveys, quasi-inertial optical reference frames will however soon present an accuracy that is similar to the ICRF. Consequently, future work is to tie the radio reference frame to quasar catalogues to align the positions of the optical sources to the ICRF (see, e.g., Bourda et al. 2007). Such a procedure enables the quality of the optical catalogues to be assessed and any systematic effect to be removed. Another important topic is the definition and the maintenance of an optical reference frame and the selection of a large sample of astrometric optical standards in view of future large surveys, especially for the upcoming space astrometry missions such as the NASA's SIM (Danner et al. 1999), or ESA's GAIA (Perryman et al. 2001) for which the astrometric accuracy is expected to be close to the VLBI one (Mignard 2003).

\section{Cross-identification between the SDSS and the $2 Q Z$}

The Sloan Digital Sky Survey (SDSS, Adelman-McCarthy et al. 2006) covers about one quarter of the sky, observed by a dedicated 2.5-m telescope located at Apache Point, New Mexico. Images are obtained in five broad optical bands (designated $u, g, r, i, z$ ) covering the wavelength range of the CCD response from atmospheric ultraviolet cutoff to the near infrared (see Fukugita et al. 1996, for details). The astrometric calibration (Pier et al. 2003) yields an accuracy per coordinate of 45 mas when reduced against the USNO CCD Astrograph catalogue (UCAC) and 75 mas when reduced against Tycho-2. The SDSS data release 5 yields some 215 millions objects (most of them are stars) and is available through SQL query at http: // wWw. sdss . org/dr5. 
The 2-degree Field Quasar Redshift Survey (Croom et al. 2004), referred to as 2QZ in the following, is based on a pre-selection of candidates from well defined criteria from a broadband $u, b_{j}, r$ colors obtained from automated plate measurements of UKST photographic plates. The magnitude of the pre-selected objects is such that $16<b_{j}<20.85$. The survey area is made of 30 fields arranged in two $75^{\circ} \times 5^{\circ}$ declination strips, one passing across the South Galactic Cap, centered on $\delta=-30^{\circ}$, and an other passing across the North Galactic Cap, centered on $\delta=0^{\circ}$. A spectroscopic follow-up of quasar candidates selected using photometric criteria is completed by a $\chi^{2}$ minimization technique to fit each spectrum to a number of quasars, galaxies, and stellar templates, with redshifts determination for extragalactic objects.

The 2QZ astrometry is discussed by Smith et al. (2005). As for the SDSS, the astrometry assessment is made against stellar catalogues and at bright magnitudes. To investigate the astrometric quality at the magnitude level of the quasars, an efficient method is to analyze the difference in coordinates for the common sources, i.e., those which were cross-identified between the SDSS and the 2QZ. To achieve this, several recognition thresholds were first tested. It appears that the number of common sources, that reaches 2053 quasars, does not change when the threshold increases from $1^{\prime \prime}$ to $2^{\prime \prime}$. This proves that all positions are accurate at the sub arc second level. This is clearly shown in Fig. 1 where we remark that a majority of objects are included in a $0.5^{\prime \prime}$-diameter circle. Note the grid structure of the plot that is due to the lack of a supplementary digit in the coordinate determinations. We can remark that the profiles of the histograms of the coordinate differences in $\alpha \cos \delta$ and $\delta$ are close to Gaussian distributions. The maximum is reached for $\Delta \alpha \cos \delta=0.09^{\prime \prime}$ and $\Delta \delta=0.06^{\prime \prime}$, meaning that, in average, the coordinates of the SDSS quasars are larger than those of the 2QZ. Note that such differences are only representative at the faintest magnitude end of the two catalogues. The rms of the differences are $\sigma_{\Delta \alpha \cos \delta}=0.2^{\prime \prime}$ and $\sigma_{\Delta \delta}=0.2^{\prime \prime}$. These values are close to the precision of the B1.0 astrometric catalogue (Monet et al. 2003).

\section{Cross-identification between the SDSS and the VLBA Calibrator Survey}

For compact radio quasars, the distance between the physical regions responsible for the optical and radio emissions is negligible in comparison to the current optical accuracy. As the astrometric precision reaches a few milliarc seconds in the optical band and the sub milliarc second in the radio band, it enables the study of sources at different electromagnetic frequencies, especially for non-compact radio sources, active galaxies, and nearby sources, which represent a large fraction of the extragalactic astrometric reference objects.

The Goddard VLBI astrometric catalogue 2007c contains the sources of the ICRF (Ma et al. 1998), of its extension ICRFExt.2 (Fey et al. 2004), as well as the sources from the six releases of the VLBA Calibrator Survey (VCS, Beasley et al. 2002; Fomalont et al. 2003; Petrov et al. 2005, 2006, 2008; Kovalev et al. 2007) ${ }^{1}$. It totalizes 3913 sources observed at $8.3 \mathrm{GHz}$ ( $X$-band) and $2.3 \mathrm{GHz}$ ( $S$-band), which coordinates are derived from the analysis of the 27 VCS sessions with 4426 dualfrequency multi-baseline 24-h geodetic VLBI experiments. To align the celestial frame to the current ICRF, a no-net rotation

\footnotetext{
1 The Goddard VLBI astrometric catalogue 2007c is made available from the NASA Goddard Space Flight Center web resource at http://vlbi.gsfc.nasa.gov/astro
}
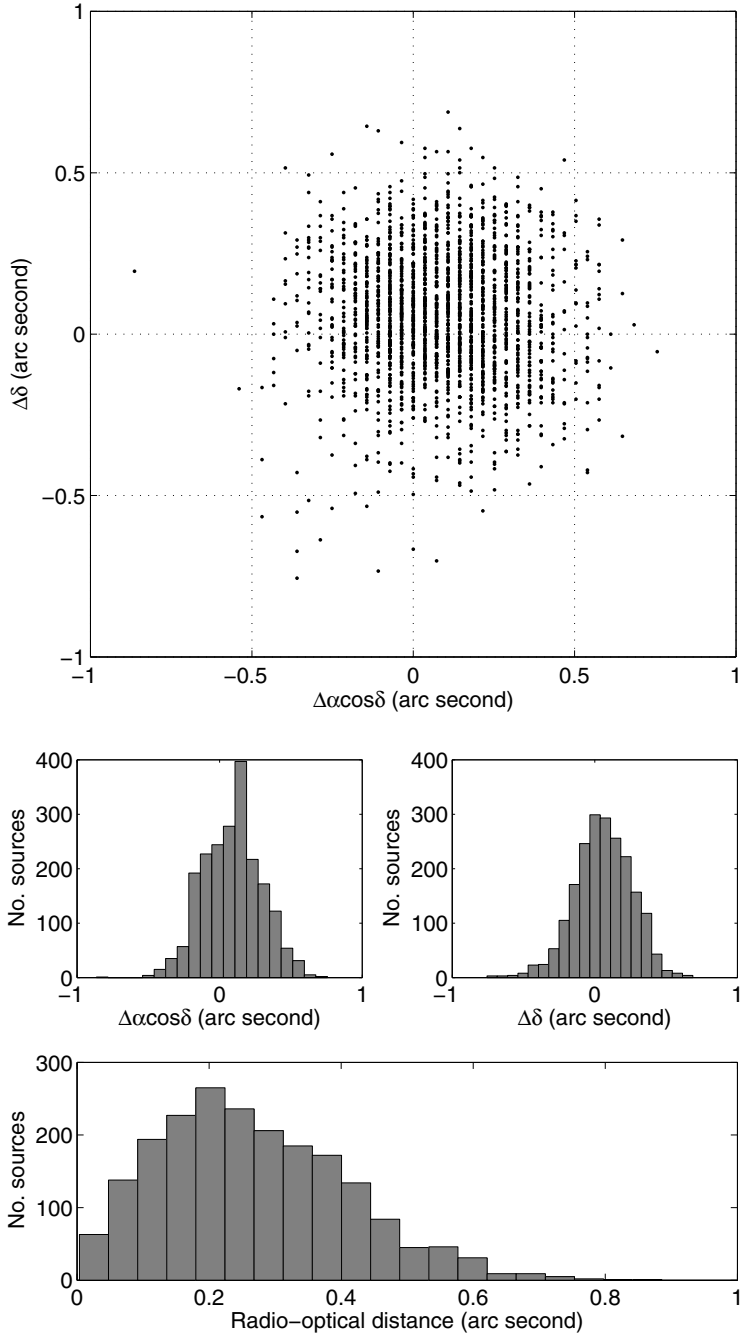

Fig. 1. Distribution of the distances between the SDSS and the 2QZ positions for the 2053 cross-identified quasars.

constraint was applied to the 212 ICRF defining sources. In this catalogue, most of the sources have a positional accuracy better than 1 mas. Some sources are flagged as having an unreliable position. We excluded them from our analysis and used the 3679 remaining sources.

As in the previous section, our cross-identification algorithm consists of checking whether an optical source in the SDSS exists within a fixed cut-off radius around a given radio source of the VCS. The cut-off radius should be similar to the astrometric precision of the less accurate catalogue. A cut-off radius of $3 \sigma$ with respect to the SDSS, let $0.23^{\prime \prime}$, seems reasonable. The comparison returns 831 sources, which is about one fifth of the complete VCS set. This value is satisfying considering that the SDSS covers one quarter of the sky. We find a mean radio-optical distance of 73 mas with a 46 mas rms. The differences between the SDSS and the VCS coordinates in declination admit an average value (12 mas) larger than in right ascension (5 mas). The bottom plot of Fig. 2 displays the radio-optical distance, showing a Poisson-like distribution peaking around 40 mas, which is of the order of the SDSS astrometric precision. The average differences show that the SDSS and the VCS catalogues can both represent the ICRF within a few mas.

We then verify whether the axes of a celestial frame defined by the optical positions of the SDSS quasars are aligned 

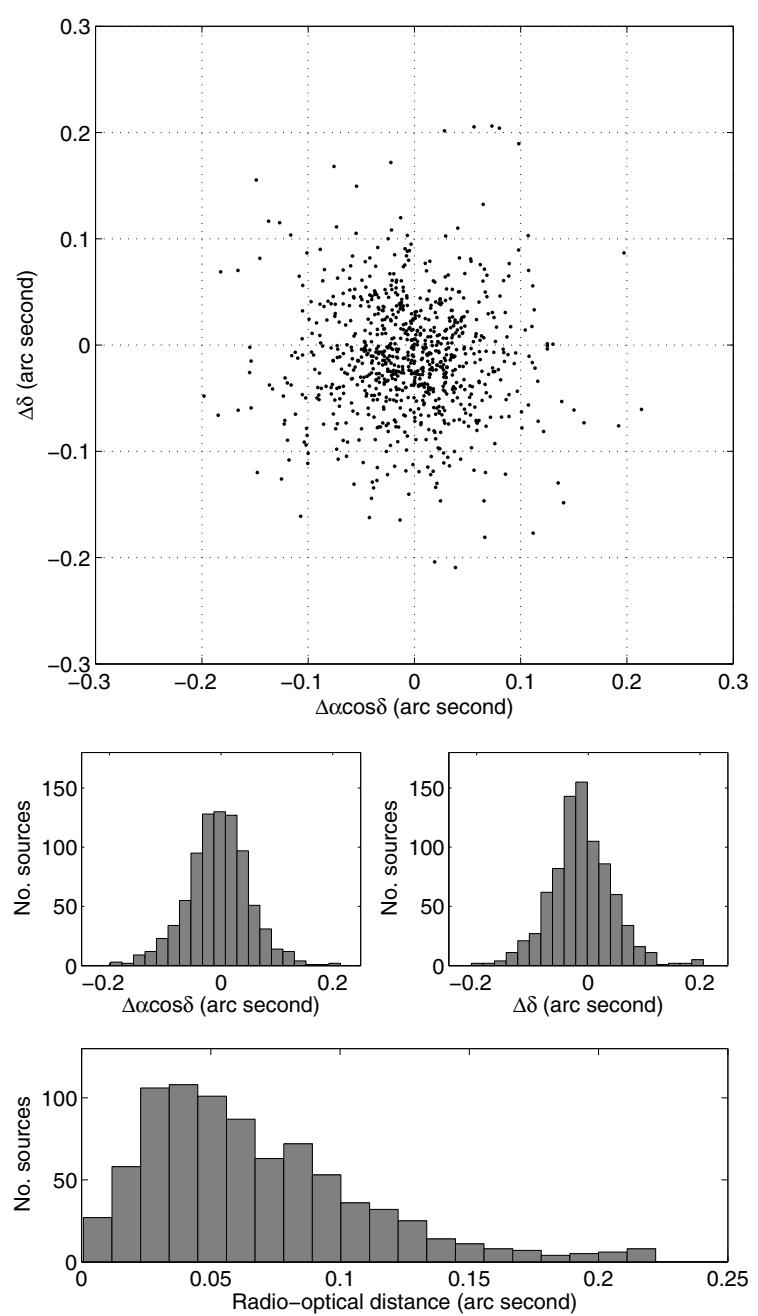

Fig. 2. Distribution of the distances between the SDSS and the VCS positions for the 831 cross-identified quasars.

with those of the VCS-defined radio reference frame (which is aligned onto the ICRF). The coordinate difference between radio and optical can be modeled by a global rotation around the three axes of the reference frame, parameterized by three angles $A_{1}$, $A_{2}$, and $A_{3}$, such that

$\Delta \alpha=A_{1} \tan \delta \cos \alpha+A_{2} \tan \delta \sin \alpha-A_{3}$,

$\Delta \delta=-A_{1} \sin \alpha+A_{2} \cos \alpha$.

These angles illustrate the consistency of the axes and poles of the celestial reference frames realized separately using radio and optical sets of source coordinates are. The estimation of the three unknown parameters is done over the cross-identified sources using a least-square fit. The difference of coordinates for each source is computed in the sense optical minus radio, so that the estimated angles of rotation are for going from the radio reference frame towards its optical counterpart. We get $A_{1}=2 \pm 3$ mas, $A_{2}=-13 \pm 3$ mas, and $A_{3}=-5 \pm 3$ mas. The significant value obtained for $A_{2}$ likely comes from an insufficient sky coverage of the SDSS than from a systematic effect on all coordinate differences. Indeed most of the SDSS sources are gathered around $\alpha=12 \mathrm{~h}$ and within $0<\delta<70^{\circ}$, leading to a correlation of -0.5 between the estimates of $A_{1}$ and $A_{3}$. In that configuration, any systematic shift in declination (due to, e.g., miscorrected atmospheric refraction) shows up in $A_{2}$.

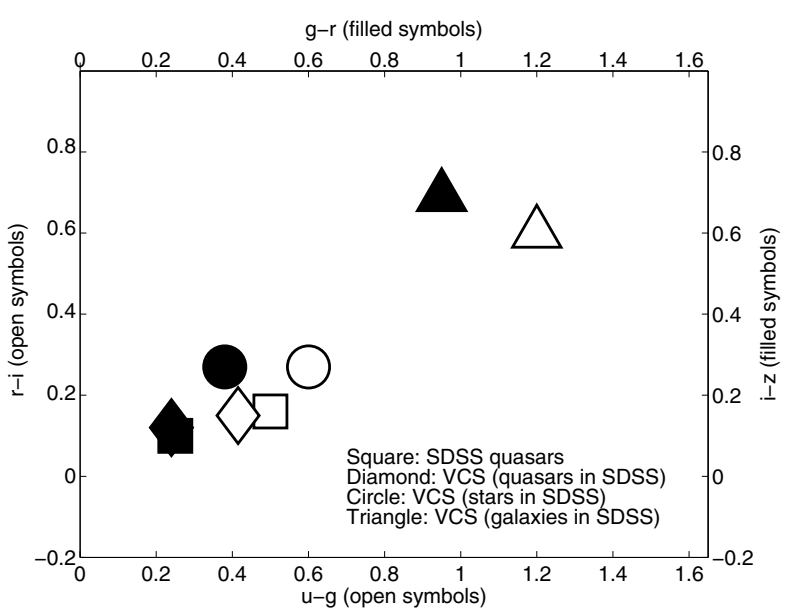

Fig. 3. Mean color locus of source category in the $u-g$ vs. $i-z$ (bottom left axes, open symbols) and $g-r$ vs. $i-z$ (upper right axes, filled symbols) planes. See comments in the text.

Note that the VLBA non uniform $(u, v)$-coverage could result in a North-South elongated beam direction for majority of the sources in the VCS. However, this issue would rather show up on the standard deviations (examination of the VCS errors shows that declinations generally have higher errors than right ascension), and at a smaller level than the SDSS precision.

Concerning the SDSS, Schneider et al. (2007) used a photometric quasar selection algorithm that retained 74429 objects intrinsically brighter than $M_{i}=-22$, assuming a cosmology consistent with the Wilkinson Microwave Anisotropy Probe (WMAP) results (Spergel et al. 2003), showing at least one emission line of $F W H M$ larger than $1000 \mathrm{~km} \mathrm{~s}^{-1}$, fainter than $m_{i}=15$ and which had a reliable determination of the redshift. The basic cosmological parameters related to this catalogue are: $H_{0}=70 \mathrm{~km} \mathrm{~s}^{-1} \mathrm{Mpc}^{-1}, \Omega_{\mathrm{M}}=0.3$ and $\Omega_{\Lambda}=0.3$. However, its appears that less than $200 \mathrm{VCS}$ sources have been caught by the algorithm, while the cross-matching of the VCS against the full SDSS database returns more than 800 sources. The forgotten objects are however listed in the SDSS database as galaxies or stars. The average unresolved and total flux density of the VLBA calibrator list is larger than the corresponding values for the VLA calibratort list. The VCS therefore represents a radio loud quasar population, whereas the SDSS sample is largely dominated by radio quiet quasars. (The latter are usually detected by optical or X-ray surveys, while the radio loud quasars are mostly detected by radio techniques and then later confirmed using optical spectroscopy.) Figure 3 displays color loci relevant to (i) the complete SDSS quasar population retained by the algorithm of Schneider et al. (squares), the VCS sources identified as (ii) quasars (diamonds); (iii) stars (circles); and (iv) galaxies (triangles) in the SDSS. The size of the symbols corresponds to the average of the standard deviation of the color differences. No standard error on the represented mean color loci is larger than 0.04. It indicates that, although the SDSS quasars, those present or not in the VCS, occupy the same loci, the VCS sources that are not flagged as quasars in the SDSS stand apart. The latter sources appear much redder, which is not surprising when considering the depletion of low redshifts quasars in the SDSS. This suggests that the redder regions of the color-color space are more suitable for identifying radio loud quasars. 


\section{References}

Adelman-McCarthy, J. K., Agüeros, M. A., Allam, S. S., et al. 2006, ApJS, 172, 634

Beasley, A. J., Gordon, D., Peck, A. B., et al. 2002, ApJS, 141, 13

Bourda, G., Charlot, P., Porcas, R., \& Garrington, S. 2007 [arXiv: 0711. 3455]

Croom, S. M., Smith, R. J., Boyle, B. J., et al. 2004, MNRAS, 349, 1397

Danner, R., Unwin, S., \& Allen, R. J. 1999, SIM: Space Interferometry Mission: Taking the Measure of the Universe (Washington, DC: NASA)

Fey, A. L., Ma, C., Arias, E. F., et al. 2004, AJ, 127, 3785

Fomalont, E., Petrov, L., McMillan, D. S., et al. 2003, AJ, 126, 2562

Fukugita, M., Ichikawa, T., Gunn, J. E., et al. 1996, AJ, 111, 1748

Kovalev, Y. Y., Petrov, L., Fomalont, E., \& Gordon, D. 2007, AJ, 133, 1236
Ma, C., Arias, E. F., Eubanks, T. M., et al. 1998, AJ, 116, 516

Mignard, F. 2003, in International Astronomical Union XXV, Joint Discussion 16: The International Celestial Reference System, Maintenance and Future Realizations, ed. R. Gaume et al., 133

Monet, D. G., Levine, S. E., Canzian, B., et al. 2003, AJ, 125, 984

Perryman, M. A. C., de Boer, K. S., Gilmore, G., et al. 2001, A\&A, 369, 339

Petrov, L., Kovalev, Y. Y., Fomalont, E., et al. 2005, AJ, 129, 1163

Petrov, L., Kovalev, Y. Y., Fomalont, E., \& Gordon, D. 2006, AJ, 131, 1872

Petrov, L., Kovalev, Y. Y., Fomalont, E., et al. 2008, AJ, submitted

Pier, J. R., Munn, J. A., Hindsley, R. B., et al. 2003, AJ, 125, 1559

Schneider, D. P., Hall, P. B., Richards, G. T., et al. 2007, AJ, 134, 102

Smith, R. J., Croom, S. M., Boyle, B. J., et al. 2005, MNRAS, 359, 57

Spergel, D. N., Verde, L., Peiris, H. V., et al. 2003, ApJS, 148, 175 